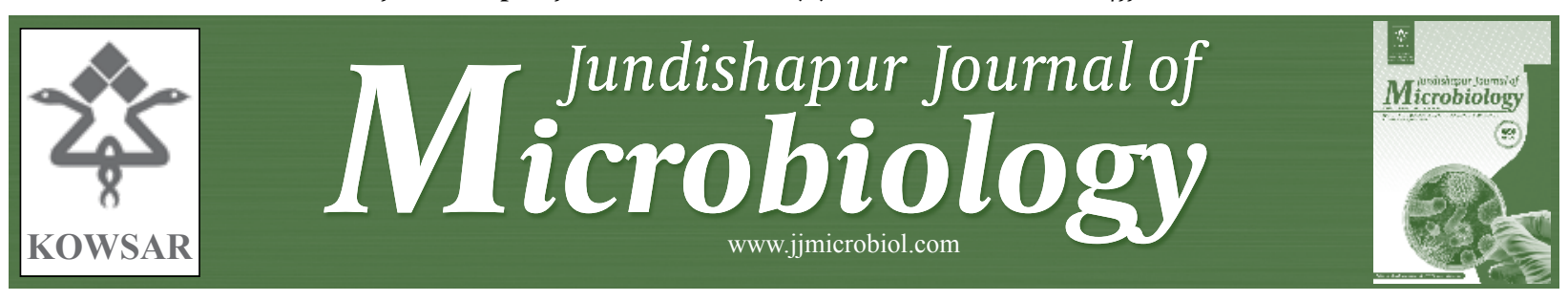

\title{
Molecular Characterization of Cryptosporidium spp. in Wild Rats of Tehran, Iran Using 18s rRNA Gene and PCR_RFLP Method
}

\author{
Fares Bahrami ${ }^{1}$, Javid Sadraei ${ }^{*}$, Mehdi Frozandeh ${ }^{2}$ \\ ${ }^{1}$ Parasitology Department of Medical School, Tarbiat Modarres University, Tehran, IR Iran \\ ${ }^{2}$ Biotechnology Department of Medical School, Tarbiat Modarres University, Tehran, IR Iran
}

\section{A R T I C L E I N F O}

Article type:

Original Article

\section{Article history:}

Received: 04 Dec 2011

Revised: 04 Feb 2012

Accepted: 06 Feb 2012

Keywords:

Cryptosporidium

Rats

PCR-RFLP

\begin{abstract}
A B S T R A C T
Background: Wild Rats have been implicated as potential reservoirs of Cryptosporidium spp., thereby responsible for transimission of the infection to humans by acting as natural sources of C. parvum, a zoonotic species. Recently, and possibly due to much closer and more frequent contacts to these animals, concerns have raised about spread of the disease among human environments.

objectives: The purpose of this study is molecular characterization of Cryptosporidium in rats of Tehran.

Materials and Methods: Rats were hunted randomly from different zones of Tehran and their stool samples were studied by Polimerase Chain Reaction (PCR) method using 18s rRNA gene. Subsequently, positive (i.e. infected) samples were characterized and sequenced.

Results: Reported rates of Cryptosporidium infection among 77 rats by implementation of staining as well as nested PCR methods were $\% 13$ and $27.3 \%$, respectively. In order to recognize type of infective agent, all positive cases were tested by PCR - RFLP (Restriction Fragment Length Polyformism) method by which C. parvum pattern was obsereved. . The obsereved patten inPCR-RFLP test was checked via sequence determination method in all positive isolations and the results were approved.

Conclusions: The results reveal that wild rats in Tehran are reservoirs of C. parvum that could be transmitted to humans. Also, it illuminates hygienic importance of freewildrats eradication throughout the city.
\end{abstract}

Copyright $\odot 2012$ Kowsar Corp. All rights reserved.

- Implication for health policy/practice/research/medical education:

Result of this study unveils relationship between wild rats, humans and domesticated animals life cycle.

Please cite this paper as:

Bahrami F, Sadraei J, Frozandeh M. Molecular Characterization of Cryptosporidium spp. in Wild Rats of Tehran, Iran Using 18s rRNA Gene and PCR_RFLP Method.Jundishapur J Microbiol. 2012;5(3):486-490. DOI: 10.5812/jjm.3580

\section{Background}

Cryptosporidium is a species of protozoan parasites that may infect digestive system tegument cells in all vertebrates. Different subspecies of these parasites, found all around the world, may illustrate different infective

* Corresponding author: Javid Sadraei, Parasitology Department of Medical School, Tarbiat Modarres University, P.O.Box 14115-331, Tehran, IR Iran. Tel: +98-2182883841, Fax:+98-2182884555, E-mail:Sadraeij@modares.ac.ir

Copyright @2012 Kowsar Corp. All rights reserved.

patterns on their hosts; some of them infect many host species (rodents and ruminants) while some may infect fewer types of hosts and some may affect only a single type. (1). Cryptosporidium spp, grouped in phylum Apicomplexa, have many commonmorphological and life cycle specifications with Coccidia $(2,3)$. Cryptosporidiosis may involve different age groups with symptoms and signs of a diarrheal infection. These organisms reproduce and grow inside gastrointestinal enterocytesbut out of cytoplasm. The disease is more severe in those who 
are suffering from immune deficiencies, either acquired or congenital. (4). Cryptosporidium hominis and C. parvum localize mostly in small intestine cells, however in some animals like rats and calves, the ileum, above cecum, is the place of choice for C. parvum. In severe immune deficient animals or humans, the parasite may also be found out of intestine. Other types such as C. muris, C. andersoni and $C$. serpentis prefer stomach mucus membrane, and . C. bailey targets respiratory Tract, or cloaca (5).

\section{Objectives}

This study was performed to demonstrate molecular characterization of Cryptosporidum in rats of Tehran, animals that have been implicated as potential reservoirs for Cryptosporidium and possible resource for infection transmission to humans because they are naturally infectedby C. parvum, a zoonotic species. Recently, some concerns about disease transmission developed, particularly in view of much closer and more frequent contacts with these animals in human environments.

\section{Materials and Methods}

In current study, 77 wild rats were hunted alive from different zones of Tehran, and transferred to the laboratory. They were anesthetized by chloroform and then killed. Collected stool samples were inserted in potassium dichromate $5 \%$ and after performing DNA extraction by QIAGEN company kit (QIAmp DNA stool mini kit) (6), nested PCR method implemented on all 77 samples..

The samples were studied by two special primers for nested PCR technique. In order to study Cryptosporidium isolations, primers with 18s rRNA gene were used. The length of produced parts with these primers were 1325bp and $825 \mathrm{bp}$ (7-9).

First stage primers PCR:

a. Forward (F1): 5'-TTCTAGAGCTAATACATGCG-3'

b. Reverse (R1): 5'-CCCATTTCCTTCGAAACAGGA-3'

Second stage primers PCR:

c. Forward (F2): 5'-GGAAGGGTTGTATTTATTAGATAAAG-3'

d. Reverse (R2): 5'- CTCATAAGG TGCTGAAGGAGTA -3'

Because of high sensitivity of characterization to PCR, nested PCR method was selected for this purpose. , .. This method consists of both primary and secondary PCR (9, 10).

Scheduled program for first stage of primary PCR by thermo cycler was :

$94^{\circ} \mathrm{C}: 5 \mathrm{~min}$

35 cycles of : $94^{\circ} \mathrm{C}: 45^{\prime \prime}, 58^{\circ} \mathrm{C}: 45^{\prime \prime}$, and $72{ }^{\circ} \mathrm{C}: 1 \mathrm{~min}$

$72{ }^{\circ} \mathrm{C}: 7 \mathrm{~min}$

$4{ }^{\circ} \mathrm{C}$ : soaking

Two enzymes, Ssp1 and Vsp1, were used to characterize genotype of the samples (Table 1).

For cutting product of second PCR, following protocol was used $(11,12)$ :

\begin{tabular}{lll}
\hline Table 1. Enzyme Features & & \\
\hline \multicolumn{4}{l}{} & Vsp 1 (AseI) & Ssp 1 \\
\hline Recognition Sequence: & ...ATTAAT... & ...AATATT... \\
& ...TAATTA... & ...TTATAA... \\
After cutting: & ...ATTAAT... & ...AATATT... \\
& ..TAATTA.. & ...TTATAA... \\
Reaction Conditions & $37^{\circ} \mathrm{C}$ & $37^{\circ} \mathrm{C}$ \\
Compatible ends & AseI, PshBI & None \\
Thermal Inactivation: & $65^{\circ} \mathrm{C}$ for 20 minutes & $65^{\circ} \mathrm{C}$ for 20 minutes \\
\hline
\end{tabular}

A mixture of $5 \mu \mathrm{L}$ of second PCR, $1 \mu \mathrm{L}$ of each enzyme, 2 $\mu \mathrm{L}$ of buffer of each enzyme was performed, followed by adding enough distilled water to make a $20 \mu \mathrm{L}$ mixture. The mixture was put in $37^{\circ} \mathrm{C}$ water bath for 2 to 24 hours. Then whole product was put in gel.

\section{Results}

After first and second PCR (Figure 1), 21 out of 77 cases (\%27.3) were reported positive with Cryptosporidium (830bp); nevertheless, infection rate of each city zone were specific to that area (Table 2).

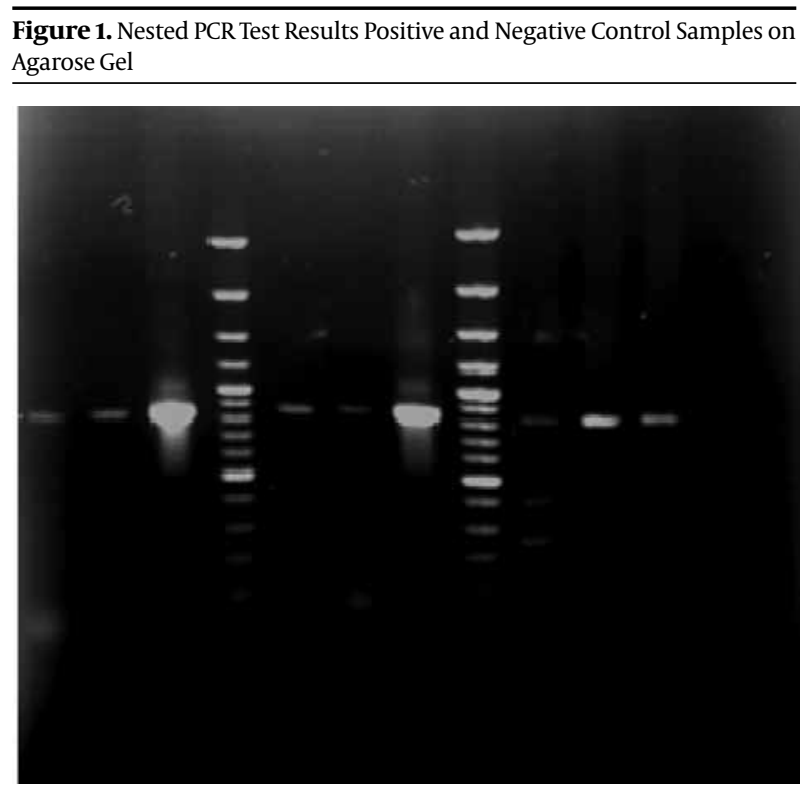

Left to right:1: Sample 33.3 South of Tehran, 2: Sample 8.1 East of Tehran, 3: Positive control sample, 4: DNA marker 100bp, 5: Sample 13.1 West of Tehran, 6: Sample 2.3 North of Tehran, 7: Positive control sample, 8: DNA marker 100bp, 9: Sample 12.1 Center of Tehran

\begin{tabular}{lll}
\hline Table 2. Results of Nested PCR Method \\
\hline Tehran Zones & $\begin{array}{l}\text { Number of Tested } \\
\text { Samples }\end{array}$ & $\begin{array}{l}\text { Results of Nested PCR } \\
\text { Test (Positives), No.(\%) }\end{array}$ \\
\hline North & 18 & $3(16.7)$ \\
South & 14 & $5(35.7)$ \\
Center & 12 & $6(50)$ \\
East & 17 & $4(23.5)$ \\
West & 16 & $3(18.7)$ \\
\hline
\end{tabular}




\subsection{The Results of Enzyme Digestion and PCR-PFLP for 18s rRNA Gene}

For type identification and genotype determination, Vsp1 and Ssp1 enzymes were used. Genotype of Cryptosporidium demonstrated 3 cutting with Vsp1 enzyme in locations 104, 115, and 629 of which 104 and 629 were visible after electrophoresis on agarose gel. With Ssp1 enzyme, cutting occurred in locations 11, 12, 108, 267, and 450 of which108, 267, and450 were visible after electrophoresis on agarose gel ( Figure 2). All 21 samples were positive in nested PCR test and were digested by Vsp1 and Ssp1 enzymes. All collected samples had RFLP results the same as Cryptosporidium parvum.This observation revealed that all Tehran rats are infected byCryptosporidiu parvium.

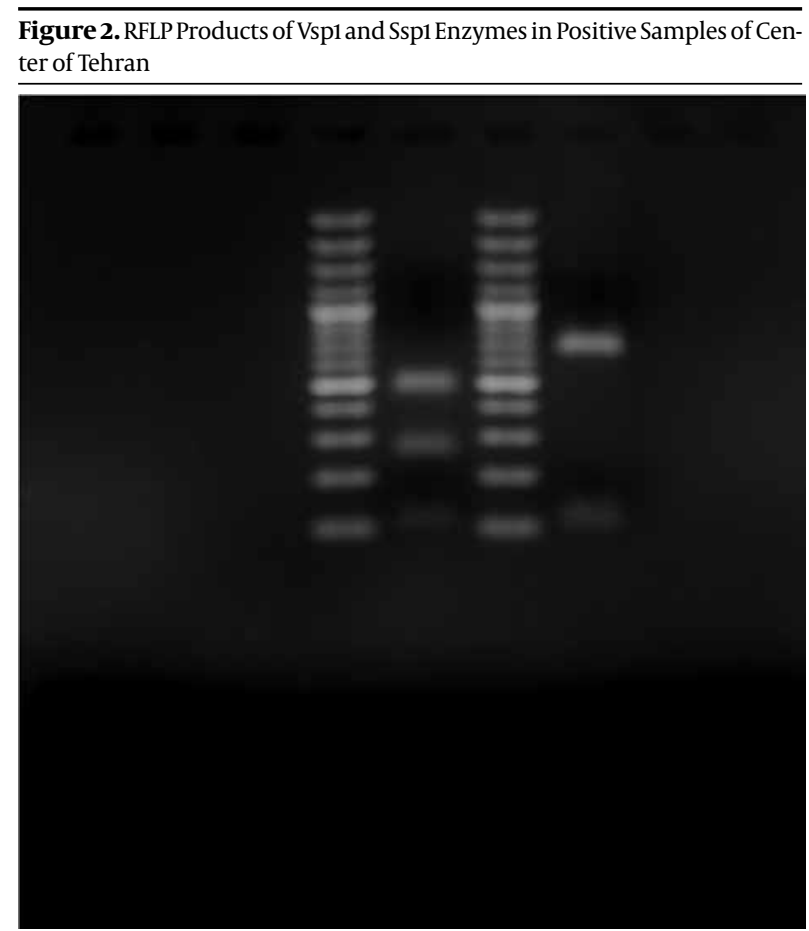

1:DNA marker 10obp, 2: Sample 22.1center of Tehran under Ssp1 enzyme activity, 3: DNA marker 100bp, 4: Sample 22.1center of Tehran under Vsp1 enzyme activity

\subsection{Results of Sequence Determination}

Following PCR-RFLP procedure on the positive samples by which Cryptosporidium type in Tehran rats were identified (Figure 3) and in order to approve such finding, sequence determination was applied on some samples. To perform this, DNA was extracted by Bioneer kits from the gel,. The results are as follows (Figure 4 and Figure5):

\subsection{Registered (HQ651732) in Gene Bank (NCBI)}

Similarities and differences of gene sequences in the samples were compared with registered Cryptosporidium genotype in Gene Bank. The result revealed that the genotypes of infective agents recognized in PCR-RFLP technique were exactly the same as for registered C. parvumgenotype .

Figure 3. RFLP Products of Vsp1 and Ssp1 Enzymes in Positive Samples of West, East, North and South Zones of Tehran

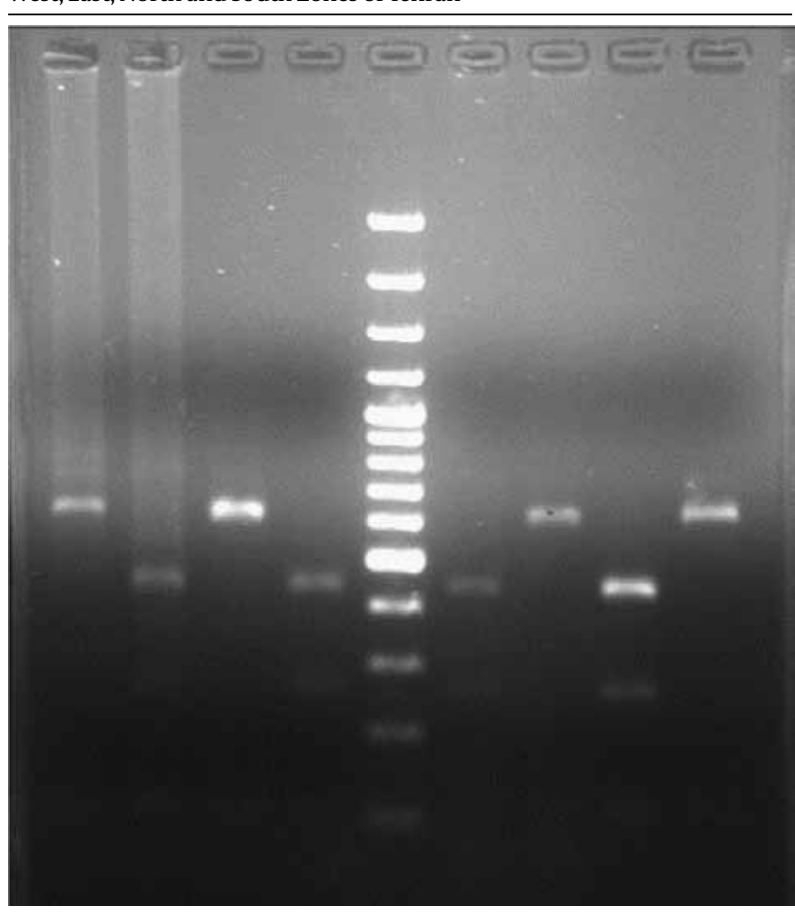

1: Sample 13.2 west of Tehran under Vsp1 enzyme activity, 2: Sample 13.2 west of Tehran under Ssp1 enzyme activity, 3: Sample 8.1 east of Tehran under Vsp1 enzyme activity, 4: Sample 8.1 east of Tehran under Ssp1 enzyme activity, 5: DNA marker 100bp, 6: Sample 28.1 north of Tehran under Ssp1 enzyme activity, 7: Sample 28.1 north of Tehran under Vsp1 enzyme activity, 8: Sample 32.1 south of Tehran under Ssp1 enzyme activity, 9: Sample 32.1 south of Tehran under Vsp1 enzyme activity

AACCAATATAATTGGTGACTCATAATAACTTTACGGATCACATTAAATG TGACATATCATTCAAGTTTCTGACCTATCAGCTTTAGACGGTAGGGTAT TGGCCTACCGTGGCAATGACGGGTAACGGGGAATTAGGGTTCGATTCC GGAGAGGGAGCCTGAGAAACGGCTACCACATCTAAGGAAGGCAGCA GGCGCGCAAATTACCCAATCCTAATACAGGGAGGTAGTGACAAGAAAT AACAATACAGGACTTTTTGGTTTTGTAATTGGAATGAGTTAAGTATAAA CCССТTTACAAGTATCAATTGGAGGGCAAGTCTGGTGCCAGCAGCCG CGGTAATTCCAGCTCCAATAGCGTATATTAAAGTTGTTGCAGTTAAAAA GCTCGTAGTTGGATTTCTGTTAATAATTTATATAAAATATTTTGATGAAT ATTTATATAATATTAACATAATTCATATTACTATATATTTTAGTATATGAAA TTTTACTTTGAGAAAATTAGAGTGCTTAAAGCAGGCATATGCCTTGAAT ACTCCAGCATGGAATAATATTAAAGATTTTTATCTTTCTTATTGGTTCTA AGATAAGAATAATGATTAATAGGGACAGTTGGGGGCATTTGTATTTAAC AGTCAGAGGTGAAATTCTTAGATTTGTTAAAGACAAACTAATGCGAAA GCATTTGCCAAGGATGTTTTCATTAATCAAGAACGAAAGTTAGGGGAT CGAAGACGATCAGATACCGTCGTAGTCTTAACCATAAACTATGCCAAC TAGAGATTGGAGGTTGTTCCTTACTCCTTCAGCACCTTATGAGAA

Figure 4. Nucleotide Sequence 824bp, Product of Nnsted PCR Isolation Number 22.1 in Center of Tehran; Registered (HQ651731) in Gene Bank(NCBI)

Results of analysis revealed that isolation number 22.1 matched $100 \%$ with C. parvum isolated from human and cow in Iran (Registration number in Gene Bank DQ656352.1 and DQ656355.1, respectively) as well as with 
CCAATATAATTGGTGACTCATAATAACTTTACGGATCACATTAAATGTG ACATATCATTCAAGTTTCTGACCTATCAGCTTTAGACGGTAGGGTATTG GCCTACCGTGGCAATGACGGGTAACGGGGAATTAGGGTTCGATTCCG GAGAGGGAGCCTGAGAAACGGCTACCACATCTAAGGAAGGCAGCAG GCGCGCAAATTACCCAATCCTAATACAGGGAGGTAGTGACAAGAAAT AACAATACAGGACTTTTTGGTTTTGTAATTGGAATGAGTTAAGTATAA ACCСCTTTACAAGTATCAATTGGAGGGCAAGTCTGGTGCCAGCAGCC GCGGTAATTCCAGCTCCAATAGCGTATATTAAAGTTGTTGCAGTTAAAA AGCTCGTAGTTGGATTTCTGTTAATAATTTATATAAAATATTTTGATGAA TATTTATATAATATTAACATAATTCATATTACTATATATTTTAGTATATGAA ATTTTACTTTGAGAAAATTAGAGTGCTTAAAGCAGGCATATGCCTTGA ATACTCCAGCATGGAATAATATTTAAGATTTTTATCTTTCTTATTGGTTC TAAGATAAGAATAATGATTAATAGGGACAGTTGGGGGCATTTGTATTTA ACAGTCAGAGGTGAAATTCTTAGATTTGTTAAAGACAAACTAATGCGG AAGCATTTGCCAAGGATGTTTTCATTAATCAAGAACGAAAGTTAGGGG ATCGAAGACGATCAGATACCGTCGTAGTCTTAACCATAAACTATGCCA ACTAGAGATTGGAGGTTGTTCCTTACTCCTTCAGCACCTTATGAGAAA.

Figure 5. Nucleotide Sequence 824bp, Product of nested PCR Isolation Number 8.3 in East of Tehran; Registered (HQ651732) in Gene Bank(NCBI)

C. parvum sub-type Sakha 212 isolated from cow milk in Egypt (Ab 513881.1). It was matched 99\% with C.parvum Isolated from human in Iran (DQ 656354.1). Isolation number 8.3 from East of Tehran matched $99 \%$ with C.parvum isolated from human and cattle in Iran (DQ656352.1 and DQ656354.1, respectively) as well as matched $98 \%$ with $C$. wrairi isolated from guinea pig (U11440.1).

\subsection{Phylogenetic Analysis}

Phylogenetic analysis was performed between sequenced isolation in this study and respective sequences of genotypes registered in Gen Bank.

In this analysis, results of sequenced isolations were arranged initially by Bio-Edit and Chromas, followed by CLUSTAL X program implementationdeveloped for alignment and drawing primary pilot tree. Subsequent- ly, genetic distance was drawn by neighbor-joining (NJ) method, the most popular and simplest method for tree drawing, and Mega4 software. Accuracy of phylogenetic tree was evaluated by boot strap re-sampling performed 1000 times (Figure 6).

By phylogenetic comparison between sequenced samples isolated from East and Center zones of Tehran, C. parvum isolated from human and cattle in Iran, and standard isolations, a close similarity might be identified among these genotypes. This similarity might be identified in lower degrees with $C$. wrairi isolated from guinea pig.

\section{Discussion}

Cryptosporidium is a protozoan of phylum Apicomplexa which can be found in all vertebrate groups around the world and may have various disease symptoms and signs. Some types of this protozoan have several hosts such as rodents and ruminants and some others have only one host (1). Cryptosporidiosis mostly manifests as a diarrheal infection in humans and may involve different age groups.

The main target area for C. hominis and C. parvum is small intestine. In some animals like rats and lambs, ileum, above cecum, is prefered site of localization for $C$. parvum. Kimora et.al (2007) studied Cryptosporidium genotype of rats in Japan. With IFA method $8 \%$, and with nested PCR $39 \%$ of rats had positive samples (13). Helmy et al (2004) found that for recognition of Cryptosporidium, microscopic method has $78.5 \%$ sensitivity and $100 \%$ specificity. This study shows that nested PCR is a more sensitive method with a simpler way of interpretation. However, it is more costly (14).Tanriverdi et al.(2003) studied C. par-

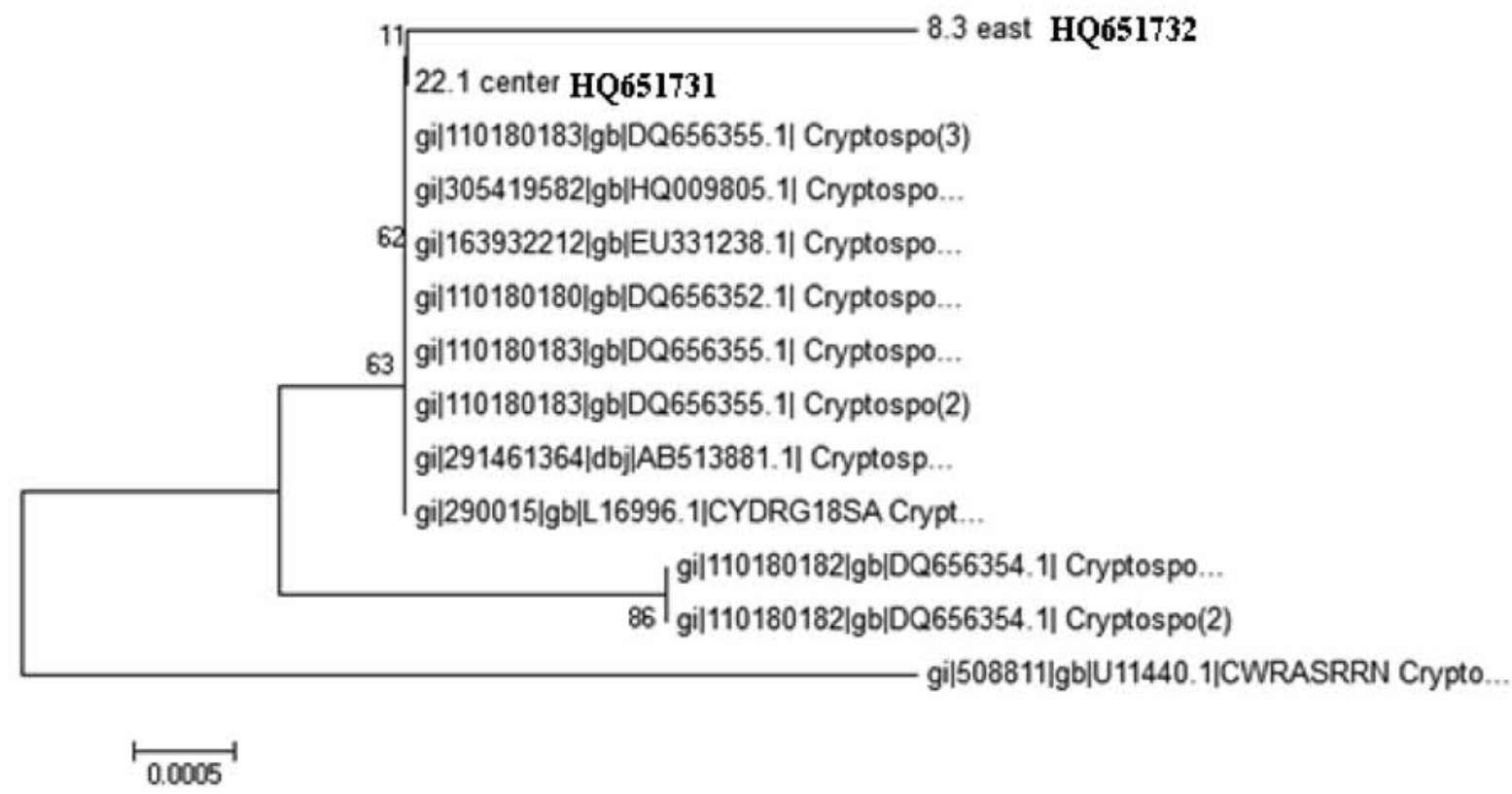

Figure 6. Phlogenetic Analysis Results of Center and East Zones of Tehran 
vum and C. hominis in human and lamb with Real-time PCR method. The results showed that not only human, who was considered as the only creature which can be infected by C. parvum, but also animals can play a role in this issue (15). Hajdušek et al. (2004) studied PCR identification of Cryptosporidium in human and animals. In their study, they used COWP and 18s rRNA genes; for type recognition and sequence determination. The study revealed that in Czech Republic the main and potential sources of this infection transmitted to humans are animals like cattle, horse and deer (16). Pirestani et al. (2008) studied the molecular determination of C. Parvum in human and cattle with 18s rRNA gene in Shahriar region. The study revealed that $C$. parvum isolated from cattle were prevailing zoonotic genotype associated with infection transmission to humans in that region (17).

Due to lack of studies about rats and Cryptosporidium infections in Iran, current study focused on Tehran rats to recognize the infection by staining and molecular methods. By using staining method, prevalence of recognized parasite was $13 \%$; it was $27.3 \%$ when nested PCR method was performed. Higher sensitivity to Cryptosporidium detection might be considered as a reason for higher prevelance figure reported by second method. . In both methods, prevalence of the infection was higher in center of Tehran than in other zones.

Considering life style in Tehran in which animals are not at risk, possibility of Cryptosporidium transmission to humans by rats increases.

Nested PCR and PCR-RFLP methods, and Ssp 1 and Vsp 1 limiting enzymes on positive samples revealed that parvum type was infective agent in all isolations. The possibility of C. Parvum transmission to humans in city zones increases when the zoonotic aspect of this infection is considered.

In sequence determination and its respective result analysis it was revealed that the $C$. parvum genotype in Tehran rats matched 99 to $100 \%$ with the C. parvum isolates of human and cattle.

By using PCR-RFLP methods, and Ssp1 and Vsp1 limiting enzymes, sequence determination and analysis of the results revealed that C. parvum in Tehran rats matched 99 to $100 \%$ with C. parvum isolates in human and cattle. The result of this study unveils the relationship between wild rats, humans and domesticated animals life cycle.

\section{Acknowledgments}

Herein, the kind cooperation of research chancellor of the university and personnel of Department of Parasitology are highly appreciated.

\section{Financial Disclosure}

\footnotetext{
The authors declare no financial disclosure.
}

\section{Funding/Support}

Funding of this work was supported by Research Chancellor of Tarbiat Modares University as M.Sc. project.

\section{References}

1. Fayer R, Ungar BL. Cryptosporidium spp. and cryptosporidiosis. Microbiol Rev.1986;50(4):458-83.

2. Carreno RA, Martin DS, Barta JR. Cryptosporidium is more closely related to the gregarines than to coccidia as shown by phylogenetic analysis of apicomplexan parasites inferred using small-subunit ribosomal RNA gene sequences. Parasitol Res. 1999;85(11):899-904.

3. Leander BS, Clopton RE, Keeling PJ. Phylogeny of gregarines (Apicomplexa) as inferred from small-subunit rDNA and beta-tubulin. Int J Syst Evol Microbiol. 2003;53(Pt 1):345-54.

4. Hunter PR, Nichols G. Epidemiology and clinical features of Cryptosporidium infection in immunocompromised patients. Clin Microbiol Rev. 2002;15(1):145-54.

5. Fleta J, Sanchez-Acedo C, Clavel A, Quilez J. Detection of Cryptosporidium oocysts in extra-intestinal tissues of sheep and pigs. Vet Parasitol.1995;59(3-4):201-5.

6. Kit QDSM. Handbook for DNA purification from stool samples. 2007.

7. Forney JR, DeWald DB, Yang S, Speer CA, Healey MC. A role for host phosphoinositide 3-kinase and cytoskeletal remodeling during Cryptosporidium parvum infection. Infect Immun. 1999;67(2):844-52.

8. Jiang J, Alderisio KA, Xiao L. Distribution of cryptosporidium genotypes in storm event water samples from three watersheds in New York. Appl Environ Microbiol. 2005;71(8):4446-54.

9. Xiao L, Escalante L, Yang C, Sulaiman I, Escalante AA, Montali RJ, et al. Phylogenetic analysis of Cryptosporidium parasites based on the small-subunit rRNA gene locus. Appl Environ Microbiol. 1999;65(4):1578-83.

10. Xiao L, Limor J, Bern C, Lal AA. Tracking Cryptosporidium parvum by sequence analysis of small double-stranded RNA. Emerg Infect Dis. 2001;7(1):141-5.

11. Gatei W, Greensill J, Ashford RW, Cuevas LE, Parry CM, Cunliffe NA et al. Molecular analysis of the $18 \mathrm{~S}$ rRNA gene of Cryptosporidium parasites from patients with or without human immunodeficiency virus infections living in Kenya, Malawi, Brazil, the United Kingdom, and Vietnam. J Clin Microbiol. 2003;41(4):1458-62.

12. Homan W, van Gorkom T, Kan YY, Hepener J. Characterization of Cryptosporidium parvum in human and animal feces by singletube nested polymerase chain reaction and restriction analysis. Parasitol Res. 1999;85(8-9):707-12.

13. Kimura A, Edagawa A, Okada K, Takimoto A, Yonesho S, Karanis P. Detection and genotyping of Cryptosporidium from brown rats (Rattus norvegicus) captured in an urban area of Japan. Parasitol Res. 2007;100(6):1417-20

14. Helmy MM, Rashed LA, el-Garhy MF. Molecular characterization of Cryptosporidium parvum isolates obtained from humans. $J$ Egypt Soc Parasitol. 2004;34(2):447-58.

15. Tanriverdi S, Arslan MO, Akiyoshi DE, Tzipori S, Widmer G. Identification of genotypically mixed Cryptosporidium parvum populations in humans and calves. Mol Biochem Parasitol. 2003;130(1):13-22.

16. Hajdusek O, Ditrich O, Slapeta J. Molecular identification of Cryptosporidium spp. in animal and human hosts from the Czech Republic. Vet Parasitol. 2004;122(3):183-92.

17. Pirestani M, Sadraei J, Dalimi Asl A, Zavvar M, Vaeznia H. Molecular characterization of Cryptosporidium isolates from human and bovine using 18s rRNA gene in Shahriar county of Tehran, Iran. Parasitol Res. 2008;103(2):467-72. 